\title{
FIXED POINTS OF NONEXPANSIVE CONDENSING MULTI-VALUED MAPPINGS ON METRIC SPACES
}

\author{
CHI-LIN YEN ${ }^{1}$
}

\begin{abstract}
In this paper we consider the existence of fixed points of nonexpansive condensing multi-valued mappings from a certain kind of metric space into itself; the spaces, here, are neither linear nor compact. Our result generalizes a theorem of Dotson and also a theorem of Bose and Mukherjee in some respect.
\end{abstract}

In this note we consider the existence of fixed points of nonexpansive condensing multi-valued mappings from a certain kind of metric space into itself. This result generalizes a theorem of Dotson [2] and also a theorem of Bose and Mukherjee [1] in some respect.

Let $(X, d)$ be a metric space. Denote by $2^{X}$ the set of all closed subsets of $X$ and by $H$ the Hausdorff metric on $2^{X}$ induced by metric $d$.

Definition 1. If $F=\left\{f_{A} \mid A \in 2^{X}\right\}$ is a family of functions from $[0,1]$ into $2^{X}$ satisfying the following conditions:

(I) $f_{A}(1)=A$ for all $A \in 2^{X}$,

(II) there is a function $\phi:[0,1) \rightarrow[0,1)$ with

$$
H\left(f_{A}(t), f_{B}(t)\right) \leqslant \phi(t) H(A, B)
$$

for all $A, B$ in $2^{X}$ and $t \in[0,1)$,

(III) for all $A \in 2^{X}$, all $t_{0} \in[0,1]$,

$$
\lim _{t \rightarrow t_{0}} H\left(f_{A}(t), f_{A}\left(t_{0}\right)\right)=0,
$$

and for all $t \in[0,1]$,

(IV) $f_{A}(t) \subset f_{B}(t)$ for all $A, B$ in $2^{X}$ with $A \subset B$, and

(V) $f_{A}(t)$ is compact whenever the set $A$ is finite; we call $F$ an $(S)$-convex structure on $X$.

REMARK 1. If $X$ is a starlike convex subset of a Banach space and if $x_{0}$ is a starlike center of $X$, then for each $A \subset X$, we define

$$
f_{A}(t)=(1-t) x_{0}+t A, \quad t \in[0,1]
$$

$F=\left\{f_{A} \mid A \in 2^{X}\right\}$ is an $(S)$-convex structure.

Received by the editors October 9, 1979 and, in revised form, July 16, 1980, December 5, 1980 and August 18, 1981.

1980 Mathematics Subject Classification. Primary 54H25, 47H10; Secondary 54C60.

1 This work was supported by NSC, Republic of China. 
Let $\gamma$ denote the measure of noncompact sets on $X$ [4], that is, for each bounded subset $A$ of $X$,

$$
\gamma(A)=\inf \{r>0 \mid A \text { can be covered by finitely many balls of radii } \leqslant r\} .
$$

Definition 2. Let $T: X \rightarrow 2^{X}$.

(1) $T$ is said to be nonexpansive if for any $x \in X$ and any $y \in X$, we have

$$
H(T x, T y) \leqslant d(x, y) .
$$

(2) A continuous mapping $T$ of $(X, d)$ into $\left(2^{X}, H\right)$ is said to be condensing if for any bounded set $A \subset X$, we have

$$
\gamma(T(A))<\gamma(A)
$$

provided that $\gamma(A) \neq 0$.

Our result is the following:

TheOREM. Let $(X, d)$ be a bounded complete metric space with an $(S)$-convex structure $F$. Then every nonexpansive condensing multi-valued mapping $T$ of $X$ into $2^{X}$ has a fixed point.

Before we prove our theorem, we first need some preliminary results related to measure of noncompact sets as follows:

LEMMA 1. Let $\gamma$ be defined as before. Then

(1) $\gamma(A)=0$ if and only if $\bar{A}$ is compact,

(2) $\gamma\left(\cup_{i=1}^{n} A_{i}\right)=\max \left\{\gamma\left(A_{i}\right) \mid 1 \leqslant i \leqslant n\right\}$,

(3) $\gamma(A) \leqslant \gamma(B)$ whenever $A \subset B$,

(4) $\gamma\left((A)_{r}\right) \leqslant \gamma(A)+r$, where $r \geqslant 0$ and $(A)_{r}=\{b \in X \mid d(b, A) \leqslant r\}$,

(5) $\gamma(\bar{A})=\gamma(A)$ for all bounded subsets $A$ of $X$.

The proof of Lemma 1 is known (see Sadovskii [5]).

REMARK 2. It is easy to see that $r>\gamma(A)$ if and only if there is a finite set $G$ such that $(G)_{r} \supset A$ if and only if there is a finite set $J$ such that $H(J, A) \leqslant r$.

In order to prove our theorem we need the following three lemmas.

Lemma 2. Suppose that $F$ satisfies (II) and (III) of Definition 1. Then for $\left\{A_{n} \mid n=\right.$ $0,1,2, \ldots\} \subset 2^{X}$ and $\left\{t_{n} \mid n=0,1,2, \ldots\right\} \subset[0,1]$ with $\lim _{n \rightarrow \infty} H\left(A_{n}, A_{0}\right)=0$ and $\lim _{n \rightarrow \infty} t_{n}=t_{0}$, we have

$$
\lim _{n \rightarrow \infty} H\left(f_{A_{n}}\left(t_{n}\right), f_{A_{0}}\left(t_{0}\right)\right)=0
$$

Proof. Since the triangle inequality is valid for the Hausdorff metric and (II) we have

$$
\begin{aligned}
H\left(f_{A_{n}}\left(t_{n}\right), f_{A_{0}}\left(t_{0}\right)\right) & \leqslant H\left(f_{A_{n}}\left(t_{n}\right), f_{A_{0}}\left(t_{n}\right)\right)+H\left(f_{A_{0}}\left(t_{n}\right), f_{A_{0}}\left(t_{0}\right)\right) \\
& \leqslant H\left(A_{n}, A_{0}\right)+H\left(f_{A_{0}}\left(t_{n}\right), f_{A_{0}}\left(t_{0}\right)\right)
\end{aligned}
$$

let $n$ tend to $\infty$; it follows from (III) and the hypothesis that we have

$$
\lim _{n \rightarrow \infty} H\left(f_{A_{n}}\left(t_{n}\right), f_{A_{0}}\left(t_{0}\right)\right)=0 .
$$


In the rest of this paper we assume that $F$ is an $(S)$-convex structure on the metric space $(X, d)$.

LemMa 3. For $0 \leqslant t \leqslant 1$, the mapping $x \rightarrow f_{\{x\}}(t)$ is nonexpansive from $X$ into $2^{X}$. Moreover, for all $A \in 2^{X}$ and $t \in[0,1]$

$$
\gamma(A) \geqslant \gamma\left(f_{A}(t)\right) .
$$

Proof. Due to (II) it is easy to see that the mapping $x \rightarrow f_{\{x\}}(t)$ is nonexpansive.

Next, if $r>\gamma(A)$, by Remark 2 there is a finite set $G$ such that $H(G, A) \leqslant r$. By (V), $f_{G}(t)$ is compact, thus for any $c>0$ there is a finite set $J$ with $H\left(J, f_{G}(t)\right)<c$. By the triangle inequality and (II) we have

$$
H\left(J, f_{A}(t)\right) \leqslant H\left(J, f_{G}(t)\right)+H\left(f_{G}(t), f_{A}(t)\right)<c+r .
$$

By Remark 2, $\gamma\left(f_{A}(t)\right)<c+r$ for all $c>0$ and $r>\gamma(A)$. Hence

$$
\gamma\left(f_{A}(t)\right) \leqslant \gamma(A) .
$$

Lemma 4. For any $A \subset X$, let

$$
g(A)=\cup\left\{f_{\bar{A}}^{-}(t) \mid 0 \leqslant t \leqslant 1\right\} .
$$

Then $\gamma(g(A))=\gamma(A)$.

Proof. It is due to (5) in Lemma 1 and the definition of $g$, we may assume that $A \in 2^{X}$. Define a real-valued function $h$ on $[0,1] \times[0,1]$ as follows:

$$
h(s, t)=H\left(f_{A}(s), f_{A}(t)\right) .
$$

It follows from condition (III) that $h$ is a continuous function on $[0,1] \times[0,1]$, and thus $h$ is uniformly continuous. In particular, for $\varepsilon>0$ there is a positive number $\delta$ such that

$$
h(s, t)<\varepsilon \text { for all } 0 \leqslant s, t \leqslant 1 \text { and }|s-t|<\delta .
$$

Hence for all $0 \leqslant s, t \leqslant 1$ and $|s-t|<\delta$ we have $f_{A}(s) \subset\left(f_{A}(t)\right)_{\varepsilon}$. Take $P=\left\{t_{0}=\right.$ $\left.0<t_{1}<t_{2}<\cdots<t_{n}=1\right\}$ be a partition of $[0,1]$ with

$$
t_{i}-t_{i-1}<\delta \text { for all } i=1,2, \ldots, n,
$$

then $g(A) \subset \cup_{i=1}^{n}\left(f_{A}\left(t_{i}\right)\right)_{\varepsilon}$.

From Lemmas 1 and 3, we have

$$
\begin{aligned}
\gamma(g(A)) & \leqslant \gamma\left(\bigcup_{i=1}^{n}\left(f_{A}\left(t_{i}\right)\right)_{\varepsilon}\right) \\
& =\operatorname{Max}\left\{\gamma\left(\left(f_{A}\left(t_{i}\right)\right)_{\varepsilon}\right) \mid i=1,2, \ldots, n\right\} \\
& \leqslant \gamma(A)+\varepsilon .
\end{aligned}
$$

Therefore, $\gamma(g(A)) \leqslant \gamma(A)$. The fact that $A \subset g(A)$ implies

$$
\gamma(g(A))=\gamma(A) \text {. }
$$

REMARK 3. It follows from (IV) and the definition of $g$ that for $A \subset B \subset X$ we have

$$
f_{\bar{A}}^{-}(t) \subset f_{\bar{B}}^{-}(t) \text { for all } t \in[0,1]
$$


and then

$$
g(A) \subset g(B) .
$$

Now we are going to prove our theorem.

PROOF OF THE THEOREM. Let $x_{0} \in X$. We denote $O_{x_{0}}$ as the set

$$
\cup\left\{(g T)^{n} x_{0} \mid n=0,1,2, \ldots\right\}
$$

where $(g T)^{0} x_{0}=\left\{x_{0}\right\}$ and $(g T)^{n} x_{0}=g\left(T\left((g T)^{n-1} x_{0}\right)\right)$ for $n=1,2, \ldots$. Since $T(g T)^{n} x_{0} \subset(g T)^{n+1} x_{0}$, we have

$$
T\left(\bar{O}_{x_{0}}\right) \subset \bar{O}_{x_{0}} \text { and } \gamma\left(T\left(\bar{O}_{x_{0}}\right)\right) \leqslant \gamma\left(\bar{O}_{x_{0}}\right) .
$$

Moreover, by (IV) and Remark 3 that

$$
g\left(T\left(O_{x_{0}}\right)\right)=g\left(\bigcup_{n=0}^{\infty} T(g T)^{n} x_{0}\right) \supset(g T)^{k+1} x_{0}
$$

for all $k=0,1,2 \ldots ;$ hence

$$
\left\{x_{0}\right\} \cup g T\left(O_{x_{0}}\right) \supset\left\{x_{0}\right\} \cup\left(\bigcup_{n=0}^{\infty}(g T)^{n+1} x_{0}\right)=O_{x_{0}}
$$

and thus

$$
\gamma\left(\bar{O}_{x_{0}}\right)=\gamma\left(O_{x_{0}}\right) \leqslant \gamma\left(g T\left(\bar{O}_{x_{0}}\right)\right)=\gamma\left(T\left(\bar{O}_{x_{0}}\right)\right)
$$

That is,

$$
\gamma\left(\bar{O}_{x_{0}}\right)=\gamma\left(T\left(\bar{O}_{x_{0}}\right)\right) .
$$

Since $T$ is a condensing mapping, we have that $\bar{O}_{x_{0}}$ is a compact subset of $X$. Now we let $\left\{r_{n}\right\}$ be a strictly increasing sequence of positive numbers with $\lim _{n \rightarrow \infty} r_{n}=1$ and let $K=\bar{O}_{x_{0}}$ and

$$
T_{n} x=f_{T x}\left(r_{n}\right) \text { for all } x \in K .
$$

For $y \in O_{x_{0}}$ we have $y \in(g T)^{k} x_{0}$ for some $k=0,1,2, \ldots$ It follows from the definitions of $T_{n}$ and $O_{x_{0}}$ and Remark 3 that

$$
T_{n} y=f_{T y}\left(r_{n}\right) \subset g(T y) \subset(g T)^{k+1} x_{0} \subset O_{x_{0}} .
$$

Then for any $x \in K$ there is a sequence $\left\{y_{i}\right\}$ in $O_{x_{0}}$ which converges to $x$. By (II) we have

$$
H\left(T_{n} y_{i}, T_{n} x\right)=H\left(f_{T y_{i}}\left(r_{n}\right), f_{T x}\left(r_{n}\right)\right) \leqslant H\left(T y_{i}, T x\right) \leqslant d\left(y_{i}, x\right) ;
$$

hence $\lim _{i \rightarrow \infty} H\left(T_{n} y_{i}, T_{n} x\right)=0$, or $T_{n} x \subset \bar{O}_{x_{0}}=K$, and thus $T_{n}$ maps $K$ into $2^{K}$. Moreover, if $x, y$ are in $K$ we have

$$
H\left(T_{n} x, T_{n} y\right)=H\left(f_{T x}\left(r_{n}\right), f_{T y}\left(r_{n}\right)\right) \leqslant \phi\left(r_{n}\right) H(T x, T y) \leqslant \phi\left(r_{n}\right) d(x, y) ;
$$

that is, $T_{n}$ is a contraction mapping of $K$ into $2^{K}$. It is due to a theorem of Nadler [3] that $T_{n}$ has a fixed point $x_{n}$ in $K$, or $x_{n} \in f_{T x_{n}}\left(r_{n}\right)$. By the compactness of $K$ there is a 
convergent subsequence $\left\{x_{n_{k}}\right\}$ of $\left\{x_{n}\right\}$, with $y_{0}$ as its limit. Hence, by Lemma 2, we have that

$$
\lim _{k \rightarrow \infty} H\left(f_{T x_{n_{k}}}\left(r_{n_{k}}\right), f_{T y_{0}}(1)\right)=0
$$

and therefore $y_{0} \in f_{T y_{0}}(1)=T y_{0}$.

It follows from our theorem and Remark 1 that we have the following:

COROLlary. Let $X$ be a starlike convex, bounded, closed subset of a Banach space $E$. Then every nonexpansive condensing multi-valued mapping of $X$ into $2^{X}$ has a fixed point.

REMARK 4. If $X$ is compact then every continuous mapping of $X$ into $2^{X}$ is condensing, hence Dotson's theorem and its Corollary 2 are special cases of our theorem and corollary, respectively.

ACKNOWLEDGMENT. The author wishes to express his appreciation to the referee who has made a lot of valuable suggestions for this paper.

\section{REFERENCES}

1. R. K. Bose and R. N. Mukherjee, On fixed points of nonexpansive set-valued mappings, Proc. Amer. Math. Soc. 72 (1978), 97-98.

2. W. G. Dotson, Jr., On fixed points of nonexpansive mappings in nonconvex sets, Proc. Amer. Math. Soc. 38 (1973), 155-156.

3. S. B. Nadler, Jr., Multi-valued contraction mappings, Pacific J. Math. 30 (1969), 473-488.

4. B. N. Sadovskii, A fixed point principle, Funktional. Anal. i Priložen. 1 (1967), 74-76.

5. ___ Limit compact and condensing operators, Russian Math. Surveys 127 (1972), 85-155.

Department of Mathematics, National Taiwan Normal University, Taipei, Republic of ChINA 